\title{
Mayor incidencia de quistes sinoviales en muñecas hiperlaxas
}

\author{
A. S. Mirchandani, S. A. Balan, M. García-Elías \\ INSTITUT KAPLAN, \\ Cirugía de la Mano y del Miembro Superior. \\ PASEO DE LA BONANOVA, 9, $2^{o} 2^{\underline{a}}$ \\ O8022 BARCELONA \\ NOVIEMBRE 2006 \\ Correspondencia: \\ Marc Garcia-Elias \\ Institut Kaplan \\ Paseo de la Bonanova, 9, 2을 08022 Barcelona \\ Núm. Teléfono : (+34) 934178484 \\ Núm. Fax: (+34) 932110402 \\ Dirección electrónica: garciaelias@infonegocio.com
}

\begin{abstract}
El grado de laxitud articular de la mano de 34 pacientes intervenidos de un quiste sinovial de muñeca fue medido y comparado con el de un grupo control compuesto por personas de la misma edad y sexo. Todas las mediciones realizadas demostraron una mayor movilidad articular en el grupo de pacientes que en el grupo control. El test de Lichtman, que investiga la laxitud mediocarpiana, fue positivo en una mayor proporción de pacientes con gangliones que en el grupo control. Estos hallazgos sugieren la posibilidad de que la laxitud articular pueda ser un factor predisponerte en la patogénesis de los quistes sinoviales.
\end{abstract}

Palabras clave: ganglión, quiste sinovial, muñeca, laxitud, hipermovilidad.
Hand laxity of 34 patients with a history of ganglia formation was measured and compared to the laxity of an equal number of normal controls with the same gender and age. All measurements demonstrated greater mobility in the patient group, compared to the control group. Midcarpal shift test, which specifically investigates ulnar midcarpal laxity, was positive in a higher proportion of patients than in the control group. These findings suggest that hipermobility may play a role in ganglion pathogenesis.

Key words: ganglion, wrist, midcarpal laxity.

Rev. Iberam. Cir. Mano - Vol. 34 - Núm. 2 - noviembre 2006 (21-26)

\section{INTRODUCCIÓN}

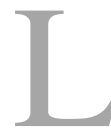

a amplitud de movimientos de una articulación en una población normal sigue una distribución del tipo Gauss, y en uno de los extremos encontramos la llamada hipermovilidad o hiperlaxitud ${ }^{1}$. Los pacientes con hiperlaxitud no solamente presentan una incidencia más alta de problemas músculo-esqueléti- $\cos ^{2}$ sino que también sufren una mayor tasa de lesiones extra-articulares, como puedan ser los prolapsos de la válvula mitral ${ }^{3}$. El llamado Síndrome de la Hipermovilidad Articular Benigna es más común en mujeres ${ }^{4}$ y se diagnostica generalmente usando los criterios de Beighton ${ }^{5}$. Estos criterios incluyen la presencia de una hiperextensión de rodilla y codo superiores a los $10^{\circ}$, hiperextensión metacarpofalángica del me- 
ñique superior a los $90^{\circ}$ y una flexión y extensión global del pulgar que permita a éste tocar el antebrazo. La hiperlaxitud localizada es más frecuente que la forma global, si bien la primera no debería ser incluida bajo este diagnóstico si no existen síntomas asociados a las hipermovilidad.

La posibilidad de que los quistes sinoviales (gangliones) sean frecuentes en personas hiperlaxas, y de que la hipermovilidad predisponga a la formación de los mismos ya fue sugerida, pero no probada, por Schernberg ${ }^{6}$. El objetivo de este estudio es determinar si existe algún tipo de relación entre ambos fenómenos, y de ser así, si esta relación es estadísticamente significativa.

\section{MATERIAL Y MÉTODOS}

La población estudiada estuvo formada por un grupo de 34 pacientes afectos de quiste sinovial con un promedio de edad de 38 años y una desviación estándar (DE) de 14 años, y de un grupo control formado por 34 individuos sanos con un promedio de edad de 38 años (DE:15). El rango de edad de ambos grupos era entre 17 y 68 años. Ambos grupos estaban compuestos por 13 hombres y 21 mujeres. En el grupo de pacientes se incluyeron personas que habían sido diagnosticadas y tratadas de un quiste sinovial (subcutáneo o intraarticular) de la muñeca o distal a la misma. Se descartaron todos aquellos pacientes cuyo problema estaba asociado a otras patologías de la muñeca o la mano. El grupo control consistió en una población de voluntarios sanos, con edades y sexo similares a los del grupo de pacientes, sin antecedentes de haber padecido gangliones ni otras enfermedades musculoesqueléticas asociadas.

Determinación de laxitud: Para cuantificar el grado de laxitud de la mano, se realizaron las siguientes mediciones:

- Distancia D1 (en milímetros): Aproximando pasivamente el pulgar hacia el antebrazo con la muñeca en extensión y desviación radial completas, se midió la distancia más corta entre el borde dorsoradial del relieve óseo del radio y el centro de la uña del pulgar (Figura 1A).
- Distancia D2 (en milímetros): Con la muñeca en máxima flexión y desviación radial y el pulgar en máxima aproximación pasiva al antebrazo, se midió la distancia más corta desde el centro de la uña del pulgar y la cara palmar del radio (Figura 1B).

- Ángulo R1 (en grados): Usando un goniómetro estándar se midió el ángulo de máxima extensión pasiva de la muñeca (Figura 1C).

- Ángulo R2 (en grados): Se midió el ángulo de máxima flexión pasiva de la muñeca (Figura 1D).

- Ángulo R3 (en grados): Ángulo de máxima extensión pasiva metacarpofalángica del dedo meñique (Figura 1E).

Todas las determinaciones, excepto las angulares, fueron normalizadas utilizando como referencia la distancia entre el borde externo de la cabeza del segundo metacarpiano y el borde interno de quinto metacarpiano.

El test de Lichtman, también denominado «Midcarpal shift» ${ }^{7}$, se realizó en todos los participantes de este estudio para determinar su grado de laxitud mediocarpiana. El test consiste en aplicar una fuerza con dirección volar sobre la muñeca relajada, en posición de flexión-extensión neutra, pronación, y máxima desviación cubital. Se define como negativo cuando la maniobra no causa ningún tipo de subluxación volar o cuando la misma sólo es posible realizando un cierto grado de fuerza. El test, en cambio, se considera positivo cuando puede mantenerse la subluxación con un mínimo de fuerza o cuando el sujeto la puede conseguir sin asistencia de un tercero. Todas las determinaciones fueron realizadas por el mismo observador y utilizando siempre los mismos instrumentos de medición. Todas las mediciones se repitieron tres veces utilizándose el promedio de las tres para este estudio.

A fin de integrar las cinco mediciones de manera que se permita una cuantificación global de la laxitud de cada individuo se utilizó el método descrito por García-Elías et al. ${ }^{8}$. Para cada una de las cinco mediciones (D1, D2, R1, R2 y R3) se otorgó a cada persona una puntuación de 0 a 20 puntos. La puntuación más alta para cada medición fue asignada a aquella persona cuyo valor para dicha medición denotaba 
A. S. MIRCHANDANI ET AL.
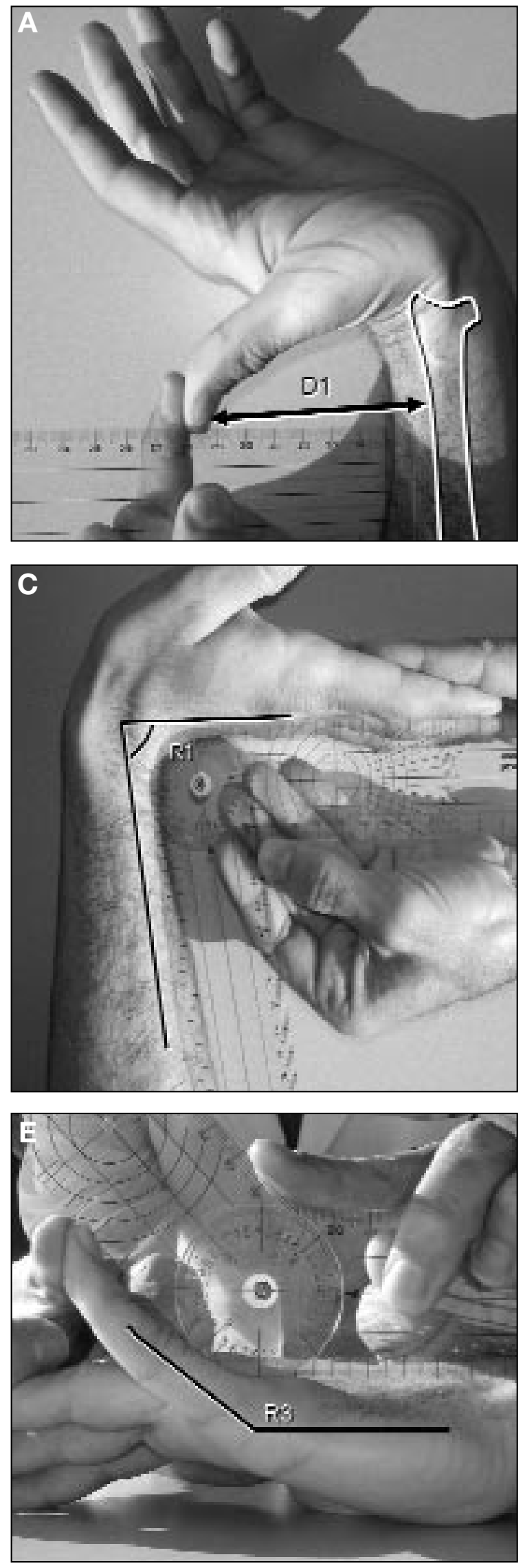
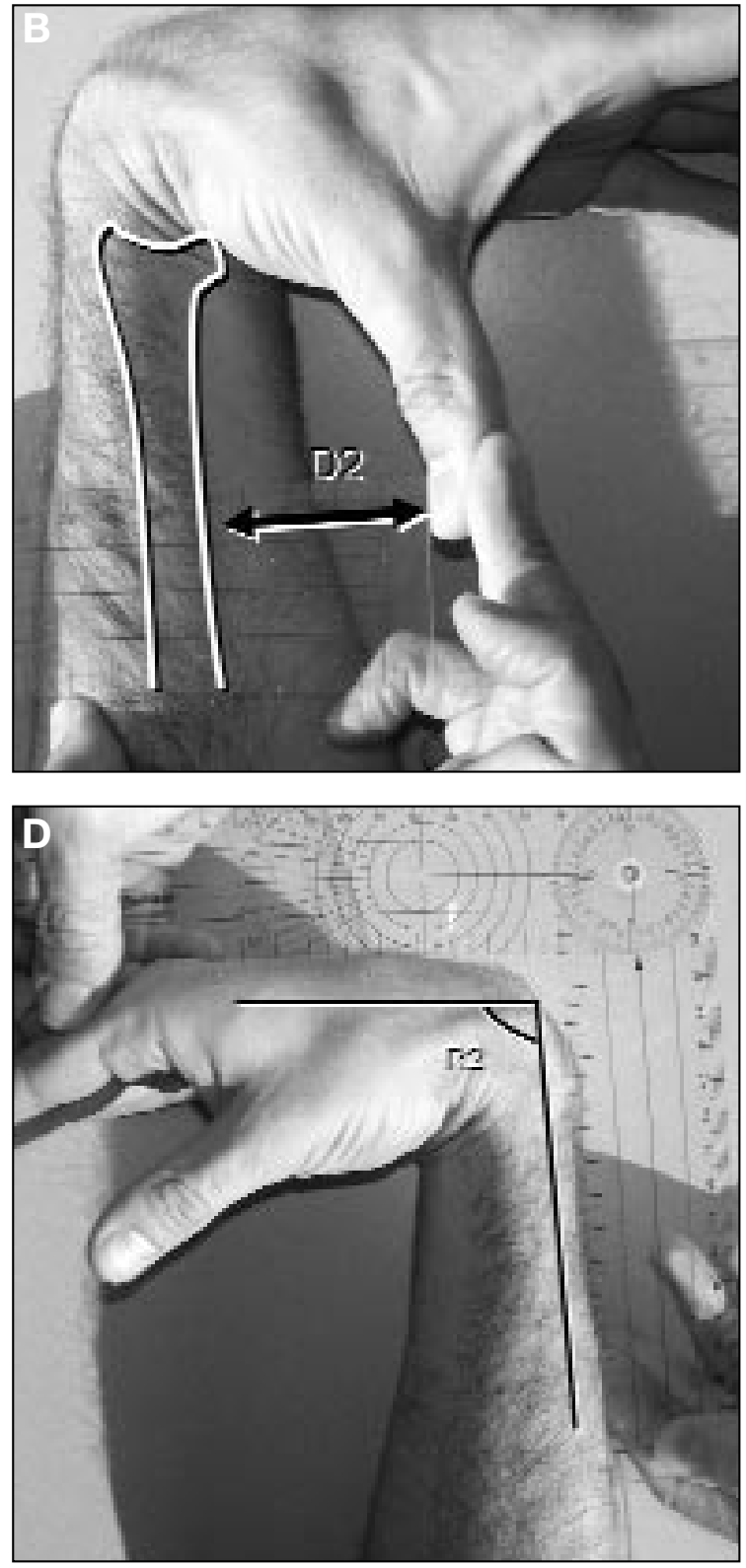

Figura 1: Maniobras clínicas utilizadas para determinar la laxitud global de la mano. A: Distancia D1: Aproximando pasivamente el pulgar hacia el antebrazo con la muñeca en extensión y desviación radial completas. B: Distancia D2: Con la muñeca en máxima flexión y desviación radial y el pulgar en máxima aproximación pasiva al antebrazo. C: Ángulo R1: Máxima extensión pasiva de la muñeca. D: Ángulo R2): Máxima flexión pasiva de la muñeca. E: Ángulo R3: Máxima extensión pasiva metacarpofalángica del dedo meñique. 
Tabla I

\begin{tabular}{|c|c|c|c|c|c|c|c|c|c|c|}
\hline & \multicolumn{2}{|c|}{$\begin{array}{l}\mathrm{D} 1 \\
\mathrm{~mm} .\end{array}$} & \multicolumn{2}{|c|}{$\begin{array}{c}\text { D2 } \\
\text { mm. }\end{array}$} & \multicolumn{2}{|c|}{$\begin{array}{c}\mathrm{R} 1 \\
\text { grados }\end{array}$} & \multicolumn{2}{|c|}{$\begin{array}{c}\mathrm{R} 2 \\
\text { grados }\end{array}$} & \multicolumn{2}{|c|}{$\begin{array}{c}\mathrm{R} 3 \\
\text { grados }\end{array}$} \\
\hline & Ganglión & Control & Ganglión & Control & Ganglión & Control & Ganglión & Control & Ganglión & Control \\
\hline Media & 50.9 & 64.1 & 63.8 & 77.9 & 89.1 & 86.2 & 86.5 & 84.4 & 67.6 & 57.8 \\
\hline D.E. & 22.3 & 19.8 & 15.4 & 12.6 & 11.8 & 12.3 & 12.1 & 9.6 & 17.7 & 19.7 \\
\hline Mín. & 0 & 22 & 26 & 53 & 70 & 58 & 64 & 62 & 18 & 15 \\
\hline Máx. & 93 & 99 & 89 & 104 & 110 & 108 & 119 & 104 & 95 & 91 \\
\hline Valor P & \multicolumn{2}{|c|}{0.03} & \multicolumn{2}{|c|}{0.0001} & \multicolumn{2}{|c|}{ No Significativo } & \multicolumn{2}{|c|}{ No Significativo } & \multicolumn{2}{|c|}{0.03} \\
\hline
\end{tabular}

D.E.: Desviación Estándar

la mayor laxitud (la distancia D1 o D2 menor, o el ángulo R1, R2 o R3 mayor), mientras que la puntuación cero fue asignada a la que demostró una medición denotando una menor laxitud (la distancia D1 o D2 mayor, o el ángulo R1, R2 o R3 menor). A las mediciones intermedias se les asignó una puntuación proporcional a los valores máximos y mínimos. Cada sujeto, por lo tanto, tuvo una puntuación total, entre 0 y 100, representando su grado de laxitud global, siendo los más laxos los que obtuvieron mayor puntuación.

Análisis estadístico: Los resultados globales obtenidos en el grupo de los pacientes, así como para cada una de las cinco mediciones, fueron comparados con los del grupo control utilizando el test de la $t$ de Student para muestras apareadas. Para la comparación de los resultados obtenidos con el test de Litchman se utilizó el método del Chi-cuadrado. Toda diferencia demostrando una $p$ ? 0.05 fue considerada estadísticamente significativa.

\section{RESULTADOS}

La laxitud global de la muñeca y la mano en el grupo de pacientes fue de 54 puntos (DE:15) mientras que para el grupo control fue de 43 puntos (DE: 15). La diferencia entre los dos grupos es significativa $(p=0.005)$. Cuando comparamos cada parámetro para ambos grupos, las diferencias obtenidas fueron significa- tivas sólo para las mediciones D1, D2 y R3. Las mediciones de movilidad pasiva de muñeca (R1 y R2) fueron mayores en el grupo de los pacientes que en el grupo control, si bien las diferencias no fueron estadísticamente significativas. (Tabla I).

El test de Litchman fue positivo en 15 de los 34 pacientes mientras que solo positivo en 7 de los 34 del grupo control $(\mathrm{p}=0.045)$.

\section{DISCUSIÓN}

Si bien la Hipermovilidad Articular Benig$n a$ no ha sido, todavía, relacionada con ningún tipo de trastorno genético, existen estudios que demuestran que las personas hiperlaxas tienen una proporción de colágeno tipo III muy superior al encontrado en la población normal ${ }^{9,10}$. Por otra parte, se ha demostrado un estrecha relación entre hiperlaxitud y una elevada incidencia de prolapso de la válvula mitral, ${ }^{3,11}$ un descubrimiento que sugiere una mayor fragilidad del colágeno tipo III que el tipo I. ¿Podría esta elevada fragilidad, en el contexto de un paciente con hipermovilidad, explicar porqué los individuos hiperlaxos desarrollan más frecuentemente degeneración mixoide de sus ligamentos? Los resultados de nuestro estudio parecen avalar esta hipótesis.

La asociación de quistes sinoviales con la hipermovilidad nos ayuda a explicar dos características de la epidemiología de los gangliones: 
1) la predisposición femenina ${ }^{12,13,14}$ y 2) la prevalencia en personas jóvenes ${ }^{15}$. Larsson ${ }^{4}$ ha demostrado que la laxitud disminuye con la edad, si bien entre las mujeres esta disminución es menos drástica que con los hombres. Lo mismo ocurre con los quistes sinoviales.

En 1990, Schernberg ${ }^{6}$ describió dos tipos de laxitud de muñeca. El tipo I se desarrolla en la articulación radiocarpiana mientras que el tipo II es mayormente una laxitud perilunar. Estudios desarrollados por García-Elías et al. ${ }^{16}$ también describen diferencias en la cinemática de los individuos laxos. Se ha visto que la presencia de laxitud perilunar altera la transmisión de cargas a través del semilunar trasladándolas a la zona periescafoidea $^{17,18}$. Parece posible que esta sobrecarga actúe como un factor predisponente en la formación de quistes sinoviales en el área escafolunar. Efectivamente, ésta es la zona donde se forman éstos con mayor frecuencia ${ }^{12,} 6$.

Los resultados del presente estudio no demuestran una diferencia estadísticamente significativa en la flexión-extensión de la muñeca de los pacientes con quistes en relación al grupo control. Probablemente, la explicación hay que buscarla en el factor muscular. Efectivamente, la resistencia muscular inconsciente a los movimientos forzados pasivos puede no haber permitido una medida del verdadero rango completo de movimiento de la articulación ${ }^{8}$. Este factor no parece ser importante con las pequeñas articulaciones como el pulgar o el dedo meñique, entendiendo que la fuerza muscular reactiva es mucho menor. El examinador en este caso puede superar fácilmente la resistencia de los músculos del antebrazo. Esto explicaría porque las medidas correspondientes al pulgar y el meñique parecen reflejar fielmente los resultados obtenidos por las puntuaciones de laxitud mientras que las de movilidad de muñeca no lo hace. En el futuro, parece deseable incluir las mediciones de la movilidad pasiva de la muñeca en el cómputo global que establece la laxitud de un individuo.

Como en la mayoría de estudios de este índole, hay que reconocer un buen número de limitaciones: 1) la población examinada es pequeña, 2) los individuos de esta muestra pertenecen racial y socialmente a la misma área geográfica, 3) las determinaciones de las medidas articulares no utilizaron elementos goniométricos sofisticados, 4) el nivel de fuerza aplicado para medir la movilidad pasiva no fue predeterminado, sino que se basó siempre en el nivel máximo de tolerancia de la persona examinada, y 5) no fue un estudio a «doble ciego» donde el examinador desconociera ni la hipótesis a demostrar ni el grupo al que pertenecía cada persona examinada. Sin embargo, las conclusiones estadísticas parecen ser concluyentes al indicar una superior incidencia del fenómeno de la hipermovilidad en el grupo de las personas afectas de quistes sinoviales en comparación con la población normal. Ciertamente, serian deseables estudios similares con mayor número de observaciones y con otras poblaciones para reforzar la validez de las evidencias obtenidas aquí.

En resumen, la hipermovilidad es mucho más que una simple curiosidad reumatológica. La hiperlaxitud de muñeca es un desorden por si mismo, con posibles consecuencias articulares, una de las cuales parece ser la predisposición a desarrollar quistes sinoviales. 


\section{BIBLIOGRAFÍA}

1. Silman AJ, Haskard D, Day S. Distribution of joint mobility in a normal population: Results of the use of fixed torque measuring devices. Ann Rheum Dis. 1986; 45: 27-30.

2. Kirk JA, Ansell BM, Bywaters EGL. The hipermobility syndrome: musculoskeletal complaints associated with generalized joint hipermobility. Ann Rheum Dis. 1967; 30:1426-30.

3. Pitcher D, Grahame R. Mitral valve prolapse and joint hipermobility: evidence for a systemic connective tissue abnormality? Ann Rheum Dis.1982; 41:352-4.

4. Larsson LG, Baum J, Mudholkar GS. Hipermobility: Features and differential incidence between the sexes. Arthritis Rheum. 1987; 30: 1426-30.

5. Beighton P, Solomon L, Soskolne CL. Articular mobility in an African population. Ann Rheum Dis. 1973; 32: 413-8.

6. Schernberg F. Roentgenographic examination pf the wrist: a systematic study of the normal, lax and injured wrist. Part
II: Stress views. J Hand Surg. 1990; 15B: 220-8.

7. Feinstein WK, Lichtman DM, Noble PC, et al. Quantitative assessment of the midcarpal shift test. J Hand Surg. 1999; 24A: 977-83.

8. Garcia-Elias M, Pitágoras T, Gilabert-Senar A. Relationship between joint laxity and radioulno-carpal joint morphology. J Hand Surg. 2003; 28B: 158-62.

9. Carver W, Nagpal ML, Nachtigal $M$, et al. Collagen expression in mechanically stimulated cardiac fibroblasts. Circulation Res. 1991; 69: 116-22.

10. Handler C, Child A, Light N, et al. Mitral valve prolapse, aortic compliance, and skin collagen in joint hypermobility syndrome. Br Heart J. 1985; 54: 501-8.

11. Grahame R, Edwards JC, Pitcher D, et al. A clinical and echocardiography study of patients with the hypermobility syndrome. Ann Rheum Dis. 1981; 40: 541-6.

12. Angelides AC, Wallace PF. The dorsal ganglion of the wrist: its pathogenesis, gross and microscopic appearance, and surgical treatment. $\mathrm{J}$ Hand Surg. 1976; 1: 228-35.

13. Dias J, Buch K. Palmar wrist ganglion: does intervention improve outcome? A prospective study of the natural history and patient reported treatment outcomes. J Hand Surg. 2003; 28B: 172-6.

14. Nelson CL, Sawmiller S, Phalen $\mathrm{G}$. Ganglions of the wrist and hand. J Bone Joint Surg. 1972; 54A: 1459-64.

15. Holm PC, Pandey SD. Treatment of ganglia of the hand and wrist with aspiration and injection of hydrocortisone. Hand 1973; 5:63-7.

16. Garcia-Elias M, Ribé M, Rodríguez J, et al. Influence of joint laxity on scaphoid kinematics. J Hand Surg. 1995; 20B: 379-82.

17. Taleisnik, J. Post-traumatic carpal instability. Clin Orthop Rel Res.1980; 149: 73-82.

18. Watson $\mathrm{KH}$, Rogers WD. A revaluation of the cause of the wrist ganglion. J Hand Surg. 1989; 14A: 812-7. 